\title{
Impact of Model-based Risk Analysis for Liver Surgery Planning
}

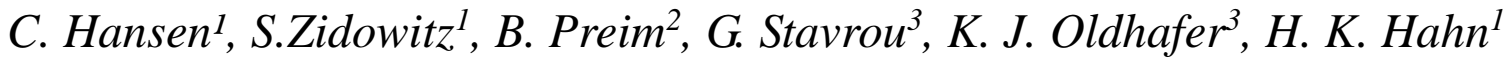 \\ ${ }^{1}$ Fraunhofer MEVIS, Institute for Medical Image Computing, Bremen, Germany \\ ${ }^{2}$ Otto-von-Guericke University Magdeburg, Faculty of Computer Science, Magdeburg, Germany \\ ${ }^{3}$ Asklepios Hospital Barmbek, Hamburg, Germany
}

\begin{abstract}
Purpose A model-based risk analysis for oncologic liver surgery was described in previous work [1]. In this paper, we present an evaluation of this method.

Methods To prove whether and how the risk analysis facilitates the process of liver surgery planning, an explorative user study with 10 liver experts was conducted. The purpose was to compare and analyze their decision making.

Results The results of the study show that model-based risk analysis enhances the awareness of surgical risk in the planning stage. Participants preferred smaller resection volumes and agreed more on the safety margins width in case the risk analysis was available. In addition, time to complete the planning task and confidence of participants was not increased when using the risk analysis.

Conclusion This work shows that the applied model-based risk analysis may influence important planning decisions in liver surgery. It lays a basis for further clinical evaluations and points out important fields for future research.
\end{abstract}

Keywords Computer-assisted surgery, Medical visualization, Liver surgery planning, Evaluation

\section{Introduction}

The determination of optimal safety margin widths around liver lesions is a challenging surgical task. Type, number, volume, and location of lesions and their relation to vessels are all important factors when deciding whether a $\mathrm{R} 0$ resection can be achieved. Thereby, surgeons have to find a compromise between the safety margin width and the estimated postoperative liver volume. 
To this end, methods for model-based risk analysis in liver surgery were developed [1-4]. We briefly describe the important steps for model-based risk analysis in liver surgery as introduced by Preim et al. (2002) [1]: vascular structures in the liver are segmented semi-automatically using a thresholdbased region-growing technique. Skeletonization with a topology-preserving thinning algorithm yields an exact centerline and vessel radii at each voxel of the skeleton. A graph analysis transforms the vessel skeleton in a directed, acyclic graph where nodes represent bi/tri-furcations. Finally, a 3D model of the vessel graph is calculated and visualized. In addition, all visible lesions and the liver surface are segmented semi-automatically and visualized in 3D.

For the visualization of vessels at risk and potentially affected liver parenchyma (territories at risk), the spatial relations between intrahepatic lesions and vessels have to be analyzed. Using an approach by Hansen et al. 2009 [2], the dependency of territories at risk from safety margins around lesions can be explored interactively. A volume-margin function visualizes the affected liver volume as a function of the safety margins width (Fig. 1, upper right). This visualization also provides information about robustness and sensitivity of vascular risk in the liver. In addition, interactive 3D renderings visualize the impaired liver volume by the portal vein (Fig. 1, upper left), and the hepatic vein (Fig. 1, upper middle).

In this work, an explorative user study was conducted to prove whether and how model-based risk analyses facilitate the process of liver surgery planning. In our previous work [1,2], this aspect was not studied in detail. The purpose of the study presented in this paper was to compare and analyze the decision making of liver surgeons and radiologic technicians.

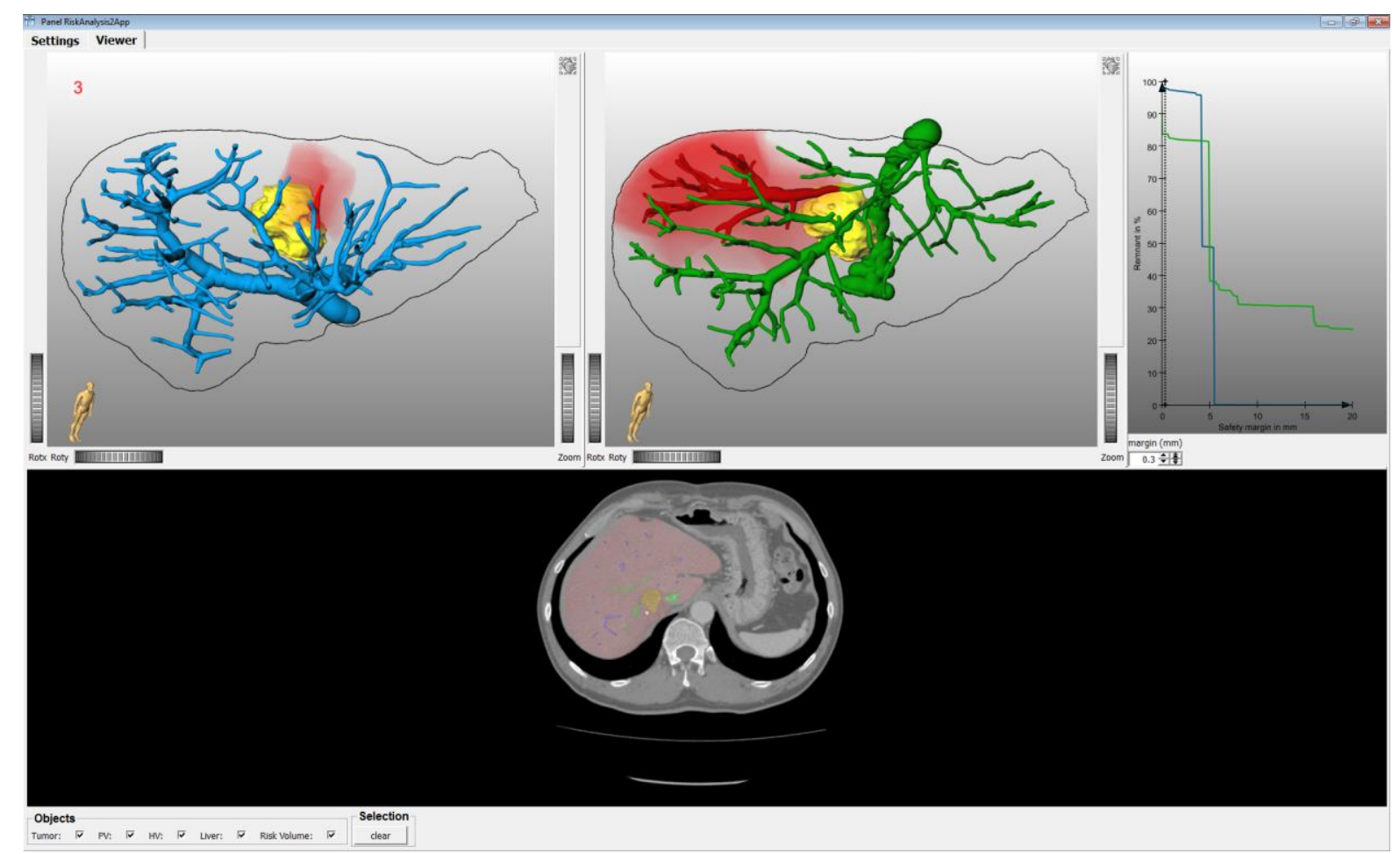

Figure 1 Screenshot from a software application for model-based risk analyses in liver surgery. The application was presented in our previous work [2]. The upper-right viewport shows two curves (hepatic vein $=$ green, portal vein $=$ blue) that visualize the remaining functional liver volume as a function of the safety margin width (volume-margin function). Territories at risk for the portal vein (blue) are visualized (red) in the upper left; for the hepatic vein (green) these territories are visualized in the upper middle viewport. The radiologic data and associated overlays of the 3D models can be accessed in the lower viewport. 


\section{Materials and Methods}

To generate evaluable data during the experiments, clinically relevant reference criteria need to be defined. These criteria should provide the basis for a comparison between the proposed method and a reference system. Three reference criteria were derived from questions that typically arise during the planning of surgical liver interventions:

(C1) resectability,

(C2) resection strategy, and

(C3) safety margins widths around lesions.

These criteria are based on subjective assessments by study participants. In addition, reference criteria which can be derived from this decision-making process are defined:

(C4) total time to analyze a case,

(C5) amount of user interaction per case, and

(C6) degree of subjective confidence in decision-making.

\section{Experiment Design}

The study consisted of two separate experiments, called experiment A and experiment B. Each participant completed both experiments. In experiment A, a reference system was presented consisting of a conventional $2 \mathrm{D} / 3 \mathrm{D}$ viewer application for planning data. While the $2 \mathrm{D}$ viewer visualizes the radiologic slice data, the 3D viewer visualizes the 3D models of vascular structures (hepatic vein, portal vein), the liver surface, and lesions. In addition, the application provides measurement tools for the assessment of distances within the dataset.

In experiment B, the utilized software application contained all functionalities that were included in the reference system. In addition, the volume-margin function of the dataset was visualized together with an interactive 3D visualization of vessels at risk and territories at risk as shown in Fig. 1.

In each experiment, participants were asked to analyze six CT datasets of the liver. The same six datasets were used in each experiment. For each dataset, participants had to perform specific planning tasks by using the software application. These planning tasks consisted of:

- determination of a virtual resection surface,

- selection of critical vessel structures which should be preserved, and

- selection of potential areas of impaired inflow and outflow.

In addition, participants completed a questionnaire for each dataset. The questionnaire directly addresses the criteria (C1-C3) defined above. In the header of each questionnaire, a report on diagnostic findings for the dataset was given and the desired postoperative liver volume was specified $(>35 \%)$.

Each experiment was conducted as follows. First, participants were informed that the experiment takes between 60 and 90 minutes and that the time is measured during the experiment. Second, the software application was presented and its graphical user interface was explained. Third, a training dataset was loaded and participants conducted the planning tasks for this dataset and filled out a questionnaire. The 
test supervisor ensured that all questions and planning tasks were understood. Finally, five test datasets were loaded in random order. Participants were informed that the experiment starts and that time is measured from now on. Analogous to the training phase, participants performed surgical planning tasks and filled out a questionnaire for each dataset. Verbal comments were transcribed during the experiment.

The experiments were performed in the context of two clinical workshops at Asklepios Clinic Barmbek, Hamburg, Germany. Because not all surgeons could take part at both workshops, several separate meetings took place. The distance between experiment A and B was always at least 3 weeks in order to minimize memory effects.

\section{Medical Datasets}

As shown in Fig. 2, our case database consisted of six abdominal CT datasets (1 training dataset, 5 test datasets). For each dataset, 3D models of the liver, hepatic vein, portal vein, and intrahepatic lesions were generated. The test datasets were selected according to the following criteria:

- presence of colorectal liver cancer,

- solitary metastases that are located adjacent to major hepatic vessels, and

- no presence of cirrhosis.

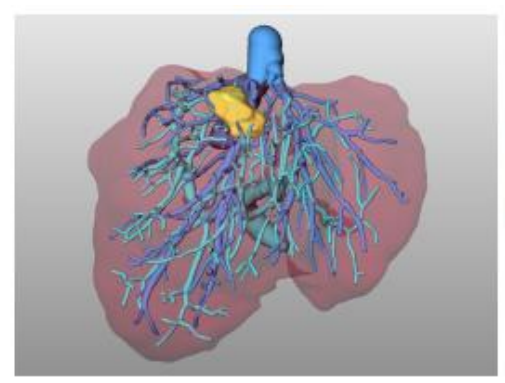

( $\mathrm{T})$

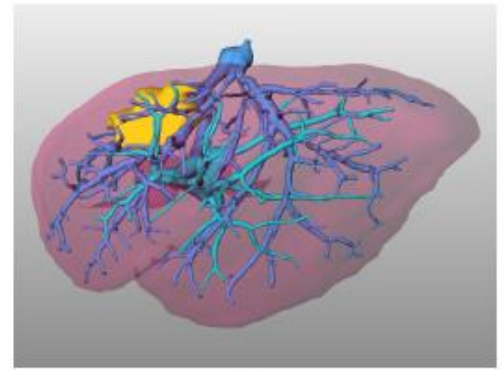

(2)

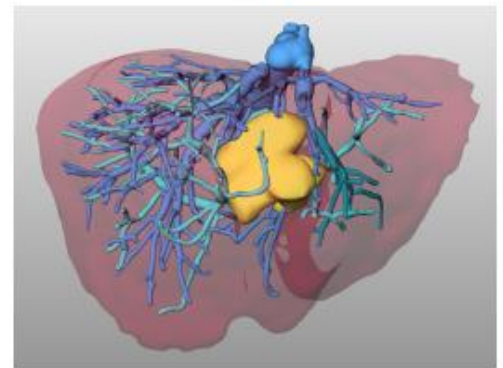

$(4)$

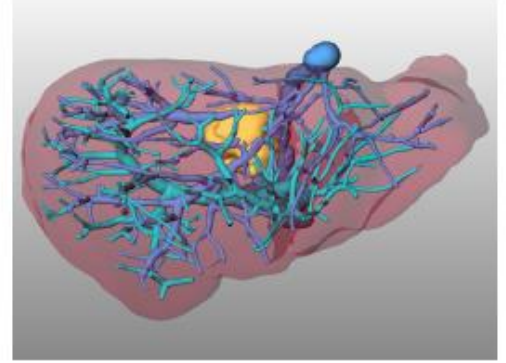

(1)

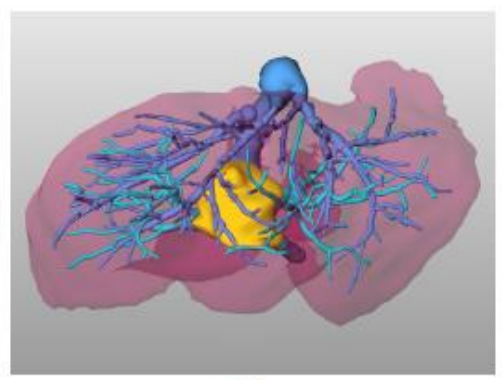

(3)

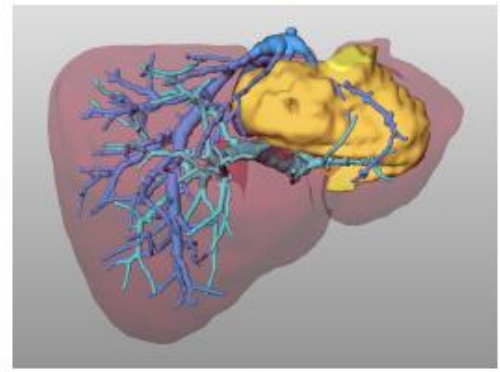

(5)

Figure 2 Selected datasets: Case $\mathrm{T}$ shows a visualization of the training dataset with a peripherally located metastasis close to the MHV. Case 1, 3, and 4 show datasets with centrally located liver metastases (yellow). In case 2 , the metastasis is located in the right liver lobe, while in case 5 it is located in the left liver lobe. 


\section{Participants}

Medical knowledge and experience in liver surgery planning are necessary to perform the planning tasks and to give meaningful answers in the questionnaire. For that reason, the subject pool consisted of 10 liver experts (three females, seven males), including four chief physicians, one senior physician, two assistant physicians, three radiology technicians. The radiology technicians were part of an expert team for liver surgery planning at MeVis Medical Solutions, Bremen, Germany. They are quite familiar in analyzing CT data of the liver and prepare virtual resection proposals for clinical customers frequently. The mean age of the participants was 41.45 years $( \pm 4.7)$. The mean number of years of surgical experience were $15.6( \pm 5.3)$, excluding the radiology technicians.

\section{Results}

Comparison of given assessments concerning patient resectability (C1) revealed that participants showed better agreement of answers in experiment B (cf. Fig. 3). In addition, the results show that participant's decisions were much more cautious and less optimistic when using the risk analysis. For example, in case 1, two surgeons who rated a patient as "resectable" in experiment A changed their decision to "non-resectable" in experiment B. Furthermore, all participants who rated a patient as "resectable with a large safety margin $>5 \mathrm{~mm}$ " in case 1,3 and 5 changed their decision to "resectable with a small safety margin $<5 \mathrm{~mm}$ ". Although there exist no significant differences between experiment A and B ( $>0.05$, Wilcoxon test), the results indicate that the proposed vascular risk analysis enhances the awareness of surgical risk in the planning stage.

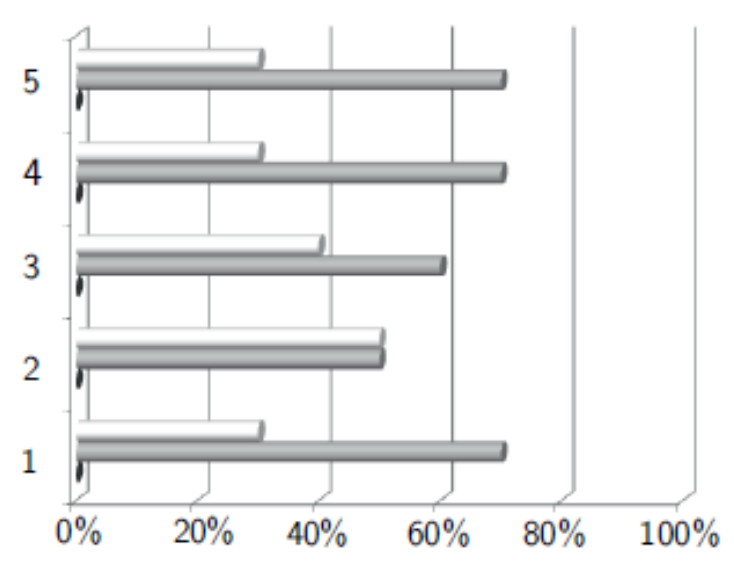

(Experiment A)

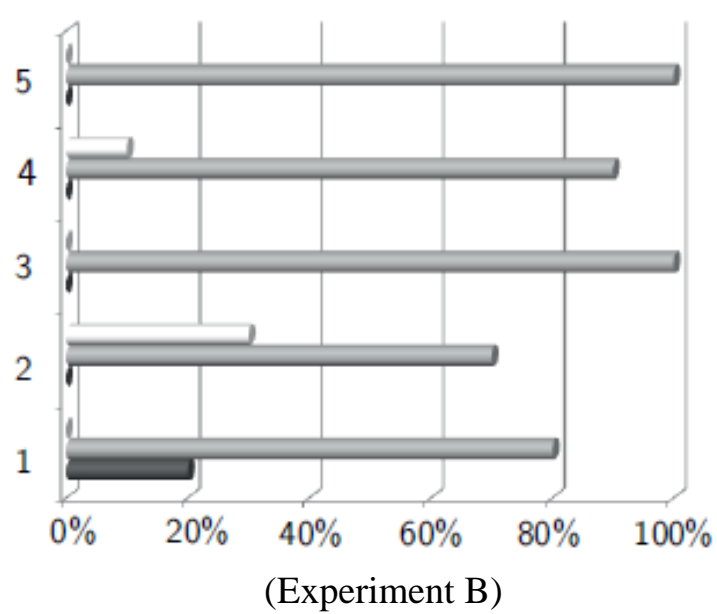

(Experiment B)

Figure 3 Assessed respectability for cases 1-5 in experiments A and B (white = "resectable with a large safety margin $(>5 \mathrm{~mm})$, light grey = "resectable with a small safety margin $(<5 \mathrm{~mm})$ ", dark grey = "not resectable").

Figures 4-6 illustrate the changes in the resection strategy (C2) that were made in the study. The amount and direction of changes reveal that the risk analysis has different magnitudes of influence on surgical decision-making that depend, among other factors, on the selected medical dataset. For cases 1 and 2, the changes show a clear trend towards the choice of smaller resection volumes when using the risk analysis. The same trend, to a lesser extent, can also be observed for cases 4 and 5. For case 3, the majority of subjects did not change their resection strategy when using the risk analysis.

The analysis shows that subjects changed their resection strategy in many cases. This is unsurprising, because it can be expected that when repeating experiment A (or B) several times with the same 
participants, the preferred resection strategy will not be constant (test-retest variability). However, the changes observed in this study follow a clear trend towards the choice of smaller resection volumes in case the risk analysis is available. This supports the above statement that the proposed methods enhance the awareness of surgical risk.

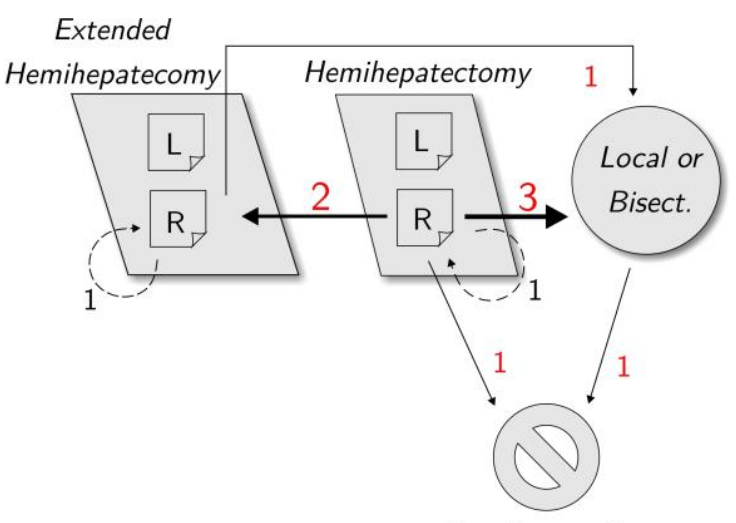

Non-Resectable

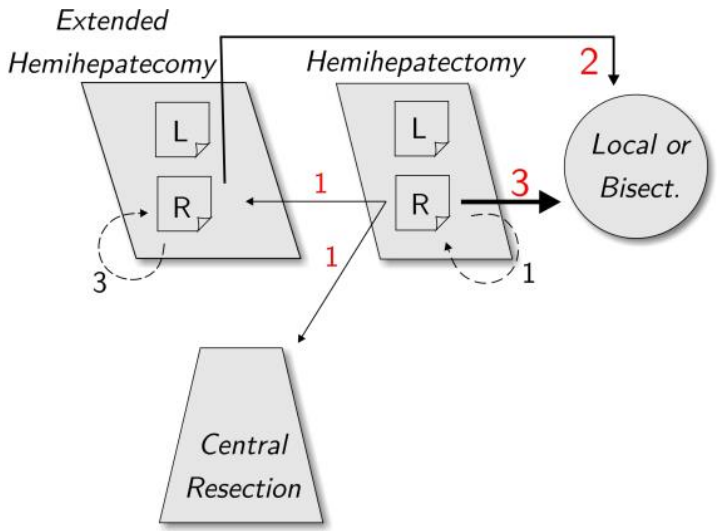

(b)

(a)

Figure 4 (a) Changes in resection strategy for case 1: While a right hemihepatectomy was the most frequently chosen resection strategy in experiment A, 6 participants changed their strategy in experiment B. The trend favors local resections $(0 / 4)$ and rejecting surgical resection $(0 / 2)$. These changes indicate that participants underestimated risk in experiment A and prefer a smaller resection volume in experiment B. (b) Changes in resection strategy for case 2: Participants show a similar trend such as in case 1. Right hemihepatectomy and extended right hemihepatectomy were found to be the most appropriate resection strategies in experiment $\mathrm{A}$. However, whereas local resections were not taken into account in experiment A, five subjects chose a local resection. Analogously to case 1 , these changes indicate that participants underestimated risk in experiment $\mathrm{A}$ and prefer a smaller resection volume in experiment $\mathrm{B}$.

Extended

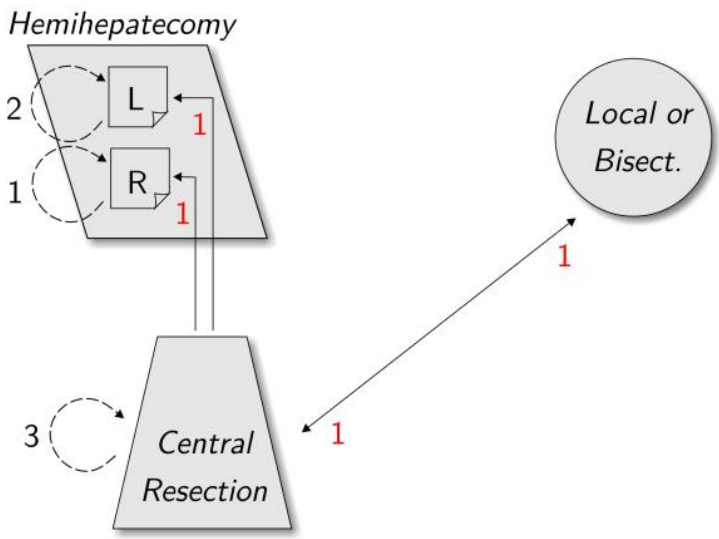

(a)

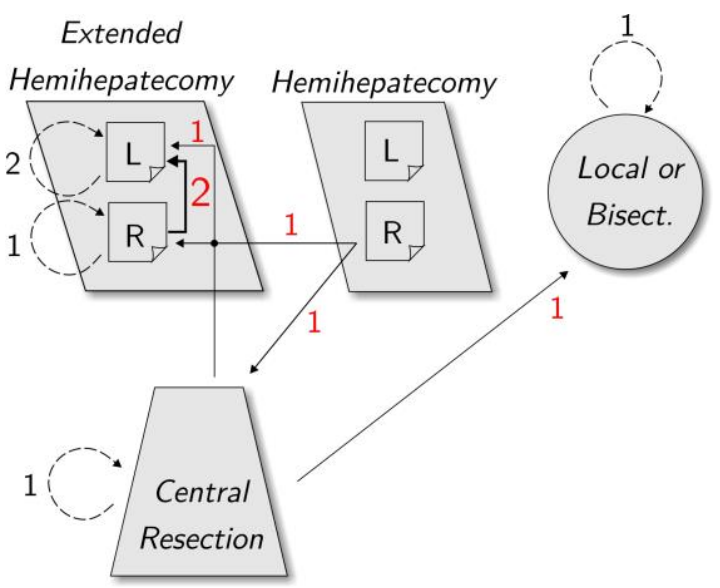

(b)

Figure 5 (a) Changes in resection strategy for case 3: The majority of subjects did not change their resection strategy. No significant trend can be observed for this case. (b) Changes in resection strategy for case 4: Several trends can be observed. First, the extended left hemihepatectomy (2/5) is the strategy most subjects preferred in experiment B, in contrast to experiment A. Second, all subjects who chose a right hemihepatectomy in experiment A changed their opinion in B. Overall, the changes indicate a trend towards smaller resection (right lobe $\rightarrow$ left lobe , central $\rightarrow$ local) when using the risk analysis. 


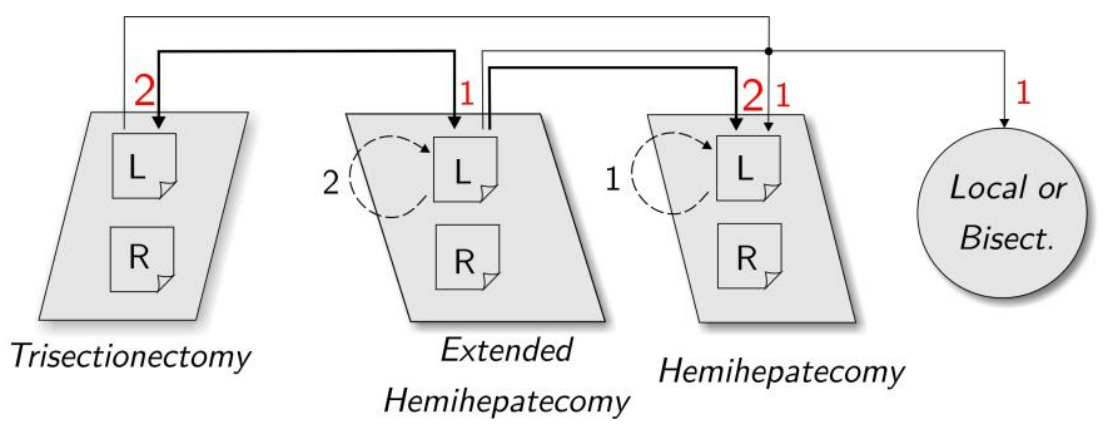

Figure 6 Changes in resection strategy for case 5: Although the strategy selections and changes of subjects seem to be inconsistent at first glance, there is a clear trend towards smaller resection volumes. 5 subjects choose a resection with a smaller resection volume than in experiment $B$.

The analysis of selected safety margins widths (C3) showed that the variation of values was lower for all cases in experiment B (cf. Fig. 7). Thus, subjects agree more when the safety margin is chosen with the proposed risk analysis (experiment B) than with 3D measurement tools (experiment A). A selected safety margin width depends on the chosen resection strategy. Thus, the measured trend to choose smaller resection volumes in experiment B seems to have an influence on the width of safety margins, or vice versa. Statistically significant differences were not found ( $p>0.05$, Wilcoxon test).

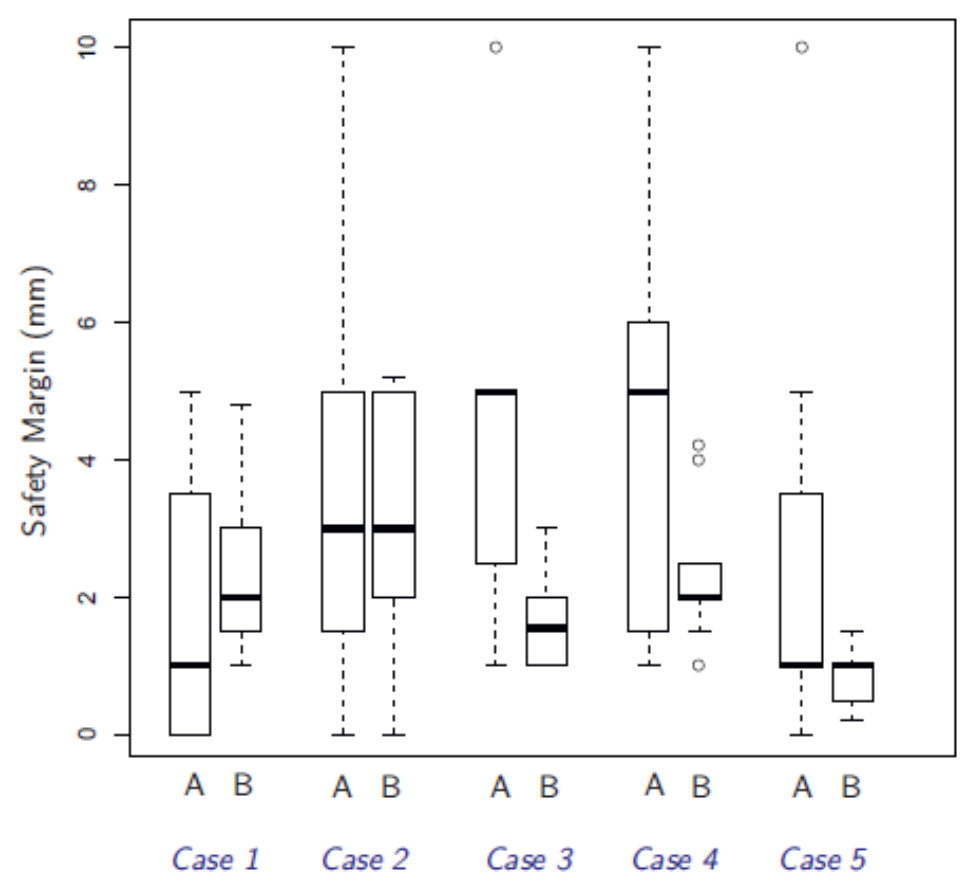

Figure 7 Selected safety margin widths for cases 1-5 in experiments A and B.

The comparison of times (C4) taken to complete the test tasks (cf. Fig. 8) revealed that there are no significant differences between experiments A and B ( $p>0.05$, Wilcoxon test). However, the way surgeons used the provided $2 \mathrm{D} / 3 \mathrm{D}$ visualization techniques was different in each experiment. In experiment A, the CT slices were more often accessed than in experiment B (cf. Fig. 9). The numbers are many times higher in experiment A, especially for cases 1 and 2.

An analysis of user interaction (C5) during the experiments also showed that the amount of interaction with the volume margin function was highest in cases 1 and 2 (cf. Fig. 10). This relation 
indicates that interaction with the $2 \mathrm{D}$ slice data is required less when the risk analysis is extensively used.

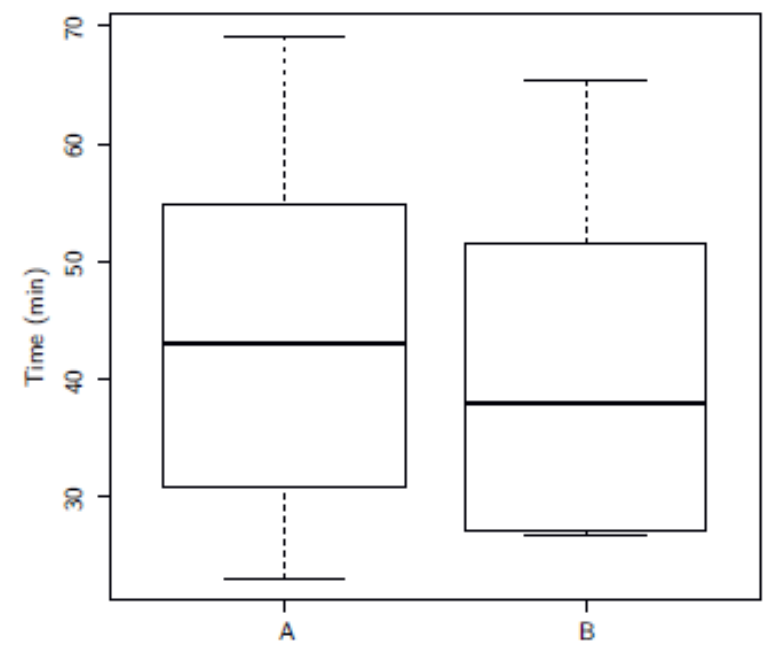

Figure 8 Time needed by participants for the completion of test tasks in experiment A and experiment B. The antennas in the box plot visualize maximum and minimum values. Data points that exceed the interquartile range more than one and a half time are defined as outliers.

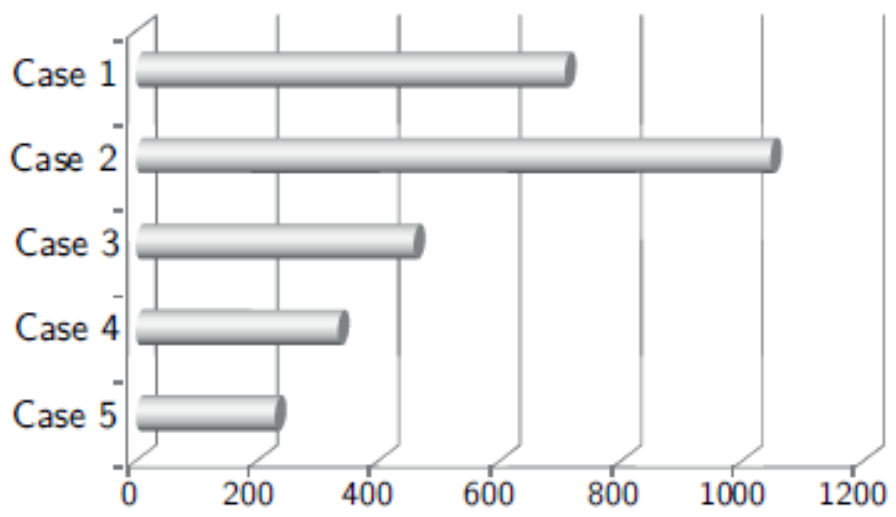

Figure 9 Number of values from the volume-margin function that were accessed in experiment B.

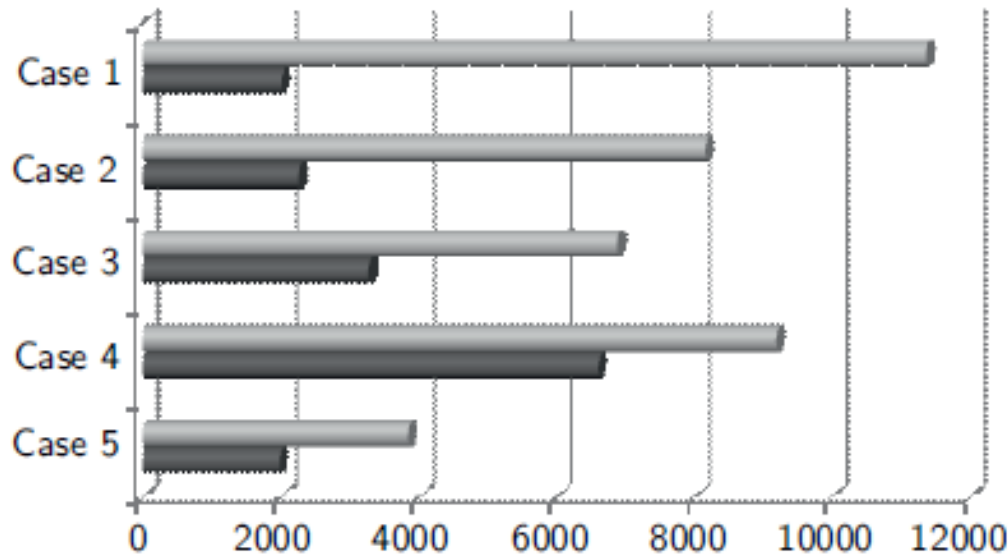

Figure 10 Total number of $\mathrm{CT}$ slices that were accessed by participants in experiment A (light grey) and experiment B (dark grey) for datasets 1-5. 
Several surgeons stated verbally that the risk analysis is especially helpful in case 1 (five surgeons), case 2 (three surgeons), and case 5 (two surgeons), because the potential areas of risk are hard to identify with 3D planning models or 2D slices. However, three surgeons noted that the territories of risk visualized for the first branching of the left portal vein in case 5 are misleading, because this part of the vessel could be saved intraoperatively by applying a special cutting approach and would therefore not represent a risk for the patient. Similar comments were also made for the inferior vena cava (extrahepatic vein with inflow of the hepatic vein). Participants suggested integrating a function that allows excluding specific vessels from the risk analyses.

The questionnaire asked participants to rank their confidence (C6) in decision-making concerning questions $2,3,5$, and 6 on an ordinal scale from 1 to $4(1=$ very sure, 2 sure, $3=$ less sure, $4=$ not sure). An analysis of the data revealed that there exist no significant differences between experiment $A$ and $B$ ( $p>0.05$, Wilcoxon test). However, the mean values indicate that participants felt more confidence in experiment A (cf. Table 1). An interesting observation in this context was that several participants mentioned that it is even more difficult to make a final decision when considering the additional information provided by the risk analysis. Two surgeons mentioned that they selected "less sure" or "not sure" in experiment B because they would prefer to discuss the resection strategy with colleagues before making a final decision. Such verbal comments were not made in experiment A.

\begin{tabular}{lcc}
\hline Question & A & B \\
\hline 2 (Resectability) & $1.9( \pm 0.63)$ & $2.2( \pm 0.75)$ \\
3 (Resection Surface) & $2.0( \pm 0.68)$ & $2.3( \pm 0.62)$ \\
5 (Resection Strategy) & $2.0( \pm 0.71)$ & $2.2( \pm 0.77)$ \\
6 (Safety Margin) & $2.3( \pm 0.63)$ & $2.4( \pm 0.63)$ \\
\hline
\end{tabular}

Table 1 Subjective confidence of participants based on a scale from 1 to 4 ( $1=$ very sure, 2 sure, $3=$ less sure, 4 $=$ not sure).

The results of the user study can be summarized as follows:

- The applied model-based risk analysis enhances the awareness of surgical risk in the planning stage (assessment of resectability, determination of resection strategy)

- Subjects prefer smaller resection volumes in case the risk analysis is available

- Subjects agree more on the safety margins width in case the risk analysis is utilized

- Subjects do not take more time when analyzing a dataset using the risk analysis. In this context, 2D slices were less accessed in case the risk analyses were available

- Confidence in decision-making is not higher when using the risk analyses

In conclusion, the results show that the methods proposed in this work facilitate the process of liver surgery planning.

\section{Discussion}

Previous studies in the field of liver surgery planning evaluated only the impact of 3D visualization [5] and virtual resection planning [6]. Thereby, the planning data was always compared with a presentation of 2D CT images. The study performed in this work investigated the usefulness of modelbased risk analysis for liver surgery planning. The results of the study show that the proposed risk analysis may influence important planning decisions for liver surgery. 
An interesting result of the study is that confidence in decision-making was not higher when using the risk analysis. The mean confidence values are even higher without the risk analysis. There are several possible explanations for this result. First, all participants were quite familiar with the 3D planning models and the exploration of 2D slice data available in experiment A. Thus, the level of trust in the new risk analyses was probably lower than in the established 2D/3D exploration techniques. This might have had an effect on the level of confidence. It is expected that the level of confidence will increase after subjects are more familiar with the approach. Second, the additional information in experiment $B$ enhanced the awareness of surgical risk and could explain why participants rated this as less confident. Thus, the subjective confidence in decision-making might correlate with risk awareness of subjects.

The mean time to complete the planning tasks was not significantly lower when using the risk analysis. Because experiment A always took place before experiment $\mathrm{B}$, a potential bias in favor of experiment B in terms of time was introduced. Thus, the results should be interpreted by taking these circumstances into account. It would be interesting to measure what happens if participants would receive more training. We assume that training has a significant effect on the time users require to complete a planning task. Another reason for this result could be the increase in risk awareness that raised up new questions during the planning process. Thus, additional time was required. It is also assumed that the high difficulty of the selected cases influenced the confidence of participants and the measured time.

For the future, it would be desirable to prove the benefit of the proposed risk analysis by evaluating them in clinical routine. This would require a clinical study with a randomized decision regarding the utilization of the results of the risk analysis and the subsequent evaluation of clinical criteria, such as complication rate, tumor recurrence, and blood loss [7]. The participants could be divided in different groups depending on their level of training (surgical experience, experience with 3D planning software and experience with model-based risk analysis) to evaluate how this affects the outcome of surgical decisions. Such separate assessments were not meaningful in the recent study due to the small number of participants. In addition, it would be important to compare preoperative resection plans with intraoperative performed resection surfaces quantitatively. Therefore, the performed resection needs to be measured intraoperatively, e.g., by using a surgical navigation system, or acquired using postoperative imaging.

In conclusion, this work contributes to computer-assisted liver surgery planning. It lays a basis for further developments and evaluations in the context of model-based risk analyses and points out promising fields for further research.

\section{References}

[1] Preim, B., Bourqain, H., Selle, D., Peitgen, H.-O., and Oldhafer, K. "Resection Proposals for Oncologic Liver Surgery based on Vascular Territories.” Proceedings of International Symposium on Computer Assisted Radiology and Surgery (CARS). Elsevier, pp. 353-358, 2002

[2] Hansen, C., Zidowitz, S., Hindennach, M., Schenk, A., Hahn, H., and Peitgen, H.-O. "Interactive Determination of Robust Safety Margins for Oncologic Liver Surgery." In: International Journal of Computer-Assisted Radiology and Surgery, 4(5), pp. 469-474, 2009

[3] Schwaiger J, Markert M, Seidl B, Shevchenko N, Doerfler N, Lueth TC. „Risk analysis for intraoperative liver surgery.“ In: Proceedings of IEEE Eng Med Biol Soc., pp. 410-413, 2010

[4] Lamata P, Lamata F, Sojar V, Makowski P, Massoptier L, Casciaro S, Ali W, Stüdeli T, Declerck J, Elle OJ,Edwin B. "Use of the Resection Map system as guidance during hepatectomy.” In: Surg Endosc., 24(9), pp. 2327-2337, 2010

[5] Lamade, W., Glombitza, G., Fischer, L., Chiu, P., Cardenas, C. E., Thorn, M., Meinzer, H. P., et al. "The impact of 3-dimensional reconstructions on operation planning in liver surgery.” In: Arch Surg, 135, pp. 
1256-1261, 2000

[6] Lang, H., Radtke, A., Hindennach, M., Schroeder, T., Fruhauf, N. R., Malago, M., Bourquain, H., Peitgen, H.-O., Oldhafer, K. J., and Broelsch, C. E. „Impact of virtual tumor resection and computer-assisted risk analysis on operation planning and intraoperative strategy in major hepatic resection.” In: Arch Surg, 140(7), pp. 629-638, 2005

[7] Schenk, A., Zidowitz, S., Bourquain, H., Hindennach, M., Hansen, C., Hahn, H. K., and Peitgen, H.-O. „Clinical relevance of model based computer-assisted diagnosis and therapy." In: Proceedings of SPIE Medical Imaging. Vol. 6915, pp. 691502-1-19, 2008 